\title{
Modeling Nonlinear Optical Properties of Transition Metal Complexes. Basis Set, Effective Core Potential, and Geometry Effects
}

\author{
Thomas R. Cundari, ${ }^{*, 1}$ Henry A. Kurtz, and Tie Zhou \\ Department of Chemistry, University of Memphis, Memphis, Tennessee 38152-6060
}

Received: December 5, 1997

\begin{abstract}
Nonlinear optical (NLO) properties of transition metal complexes are studied using quantum chemical calculations. By comparison with all electron calculations, effective core potentials have been shown to be competent for the calculation of NLO properties as long as the valence basis sets are comparable. While overall the basis set effects are important for calculation of NLO properties, they are found to be less important for the central transition metal than for the surrounding ligands. Augmenting the basis set of main group elements with diffuse $\mathrm{s}, \mathrm{p}$, and $\mathrm{d}$ functions in a proper way could provide the best compromise between speed and accuracy of the computation. Interesting trends are found in the calculation of NLO properties of $\left[\mathrm{MO}_{4}\right]^{q-}$. Both polarizability $(\alpha)$ and second hyperpolarizability $(\gamma)$ decrease toward the right across the transition series. The second series $\left[\mathrm{MO}_{4}\right]^{q-}$ have the largest $\alpha$ among the three metalates in a triad. For group IVB and VB complexes with larger charges $\left(-4\right.$ and -3 , respectively), the second series $\left[\mathrm{MO}_{4}\right]^{q-}$ have the largest $\gamma$, while for groups VIB, VIIB, and VIII, with less anionic metalates $(-2,-1$, and 0 , respectively), the third series metalates have the largest $\gamma$. The relative difference in both $\alpha$ and $\gamma$ values among the three series in the same group is much smaller than between different groups. Overall, variations in the calculated values of NLO properties correlate with $\mathrm{M}-\mathrm{O}$ bond lengths and hence the size of the metalate ion.
\end{abstract}

\section{Introduction}

Nonlinear optical (NLO) properties are of interest wherever the interaction of light and matter is important, e.g., optical computing or laser and fiber optical materials. ${ }^{2}$ NLO materials have been utilized in information processing, optical switching, optical frequency conversion, and telecommunications. ${ }^{3}$ NLO properties have been studied experimentally and computationally with more focus on organic than inorganic compounds. Recently, there has been increased interest in organometallic and coordination complexes. ${ }^{3}$ A systematic investigation of the NLO properties for families of related complexes is crucial for improved understanding of structure-property relationships and thus the design of novel NLO materials. Therefore, reliable and rapid prediction of the NLO properties of molecules and bulk materials would be helpful. Computational chemistry has been shown to be efficient for this purpose. However, only a few investigations have been reported for inorganic species. ${ }^{3}$

The main focus of this research is the computation of nonlinear optical properties, including the development of new methods and application of new and old approaches, to interesting inorganic systems: transition metal-chalcogenidos $\left(\mathrm{L}_{n} \mathrm{M}=\mathrm{Ch}: \mathrm{L}_{n}=\right.$ ancillary ligands; $\mathrm{M}=$ transition metal; $\mathrm{Ch}$ $=\mathrm{O}, \mathrm{S}, \mathrm{Se}, \mathrm{Te})$. Three effects-basis set, geometry, metal-on calculated NLO properties are of interest in the present research.

Transition metal-oxos are ideal target complexes for several reasons. First, their molecular and vibrational structure has been studied extensively. ${ }^{4-6}$ Quantum modeling has found to be excellent for these complexes.,5 There are considerable experimental data regarding their molecular structure and electronic spectra which are crucial in validating computational

* Address correspondence to this author. results. Second, previous results by Cundari et al. ${ }^{4}$ show conclusively that effective core potential (ECP) approaches are capable of very accurately predicting the geometry of diverse $\mathrm{L}_{n} \mathrm{M}=\mathrm{Ch}$ complexes. As Kanis et al. have pointed out, ${ }^{7} \mathrm{NLO}$ properties are very sensitive to geometry. Third, these systems are related to metalates, such as barium titanate and lithium niobate, that have been widely investigated by experimentalists as NLO materials. ${ }^{2}$ The chalcogen-metal charge-transfer band in their electronic spectra is thought to be associated with large optical nonlinearity.

There are two major goals for this research. First, we wish to assess the utility of ECPs for calculating NLO properties. Second, by changing metal and/or ligands we can methodically probe the effect of different chemical influences (charge, geometry, metal) on the NLO properties. In this paper, we report the initial results for the calculation of NLO properties of transition metal complexes focusing primarily on metalate, $\left[\mathrm{M}(\mathrm{O})_{4}\right]^{q-}$, complexes.

\section{Computational Method}

Effective core potentials plus valence basis sets ${ }^{8}$ augmented by diffuse $\mathrm{s}, \mathrm{p}$, and $\mathrm{d}$ functions are used to describe transition metals and main group elements in the calculation of molecular NLO properties. Specific computational details regarding basis sets, wave functions, property evaluations, etc., are extensively described in reviews. ${ }^{9,10}$ Hartree-Fock (HF) wave functions were employed. NLO calculations were carried out using the GAMESS program ${ }^{11}$ running sequential (IBM RS-6000/350) and parallel (IBM SP2 at Cornell Theory Center and SGI PowerChallenge at the National Center for Supercomputing Applications).

The time-dependent Hartree-Fock (TDHF) method implemented in the GAMESS program by Kurtz et al. ${ }^{12}$ was employed 
for all NLO calculations. The polarizability $(\alpha)$ and the second hyperpolarizability $(\gamma)$ are the static field $(\mathrm{dc})$ isotropic average values defined by ${ }^{10,12}$

$$
\langle\alpha\rangle=\left(\alpha_{x x}+\alpha_{y y}+\alpha_{z z}\right) / 3
$$

and

$$
\langle\gamma\rangle=\left[\gamma_{x x x x}+\gamma_{y y y y}+\gamma_{z z z z}+2\left(\gamma_{x x y y}+\gamma_{x x z z}+\gamma_{y y z z}\right)\right] / 5
$$

The first hyperpolarizability $(\beta)$ is not included in this study since it is always zero because the molecules calculated have a center of symmetry.

Reliable calculation of NLO properties requires more careful attention to SCF convergence than typical electronic structure calculations. ${ }^{10}$ Hence, the following GAMESS options were employed: ${ }^{11}$ ICUT $=20$ (integrals less than $10^{-20}$ are ignored; default, ICUT $=9$ ), ITOL $=30$ (products of primitives whose preexponential factor is less than $10^{-30}$ are skipped; default, ITOL $=20)$, INTTYP $=$ HONDO (HONDO/Rys integrals used for all integral evaluations, slightly more accurate but slower than the default, INTTYP $=$ POPLE), and NCONV $=10$ (convergence is reached when the absolute value of the density change between two consecutive SCF cycles is less than $10^{-10}$; default, $\mathrm{NCONV}=10^{-5}$ ).

\section{Results and Discussion}

1. Effective Core Potentials in the Calculation of NLO Properties. ECP calculations remove the chemically less important core electrons and orbitals and thus yield great savings in computer resources. Effective core potentials and valence basis sets (VBSs) ${ }^{8}$ are used for all heavy atoms. ECPs replace the innermost core (semicore) orbitals for the transition metals (TM) and all core orbitals for main group (MG) elements. Thus, the $n \mathrm{~s}, n \mathrm{p}, n \mathrm{~d},(n+1) \mathrm{s}$, and $(n+1) \mathrm{p}$ are treated explicitly for the d-block and the $n \mathrm{~s}$ and $n \mathrm{p}$ shells for the main group. In the standard implementation, TM VBSs are triple- $\zeta$ for the valence orbitals $(n \mathrm{~d},(n+1) \mathrm{s},(n+1) \mathrm{p})$, while the outermost core orbitals $(n \mathrm{~s}, n \mathrm{p})$ have a single- $\zeta$ description. MG elements have a double- $\zeta$-plus-polarization VBS. ${ }^{9}$ It has been shown that ECPs can very accurately predict the geometry and vibrational data of diverse $\mathrm{L}_{n} \mathrm{M}=\mathrm{Ch}$ complexes. ${ }^{9}$

The NLO properties for the $\mathrm{TiCl}_{4}$ molecule are calculated employing ECPs and all-electron basis sets to assess the effect of ECPs. Reported experimental $\alpha$ for inorganic complexes are rare since unlike organometallics, few form stable, molecular species under ambient conditions. Indeed, we were only able to find two for transition metal complexes: $\mathrm{TiCl}_{4}$ and $\mathrm{OsO}_{4}{ }^{13}$ Although the latter is an oxo complex, we chose the former for this comparison given the extreme difficulties in doing allelectron calculations on an element as heavy as osmium.

The experimental geometry of $\mathrm{TiCl}_{4}(\mathrm{Ti}-\mathrm{Cl}=2.170 \AA$, gas phase $)^{13}$ is used in all calculations. Computational results are shown in Table 1. It can be seen that similar NLO properties are obtained from ECPs and all-electron basis sets as long as comparable valence basis sets are used. Compared with an experimental polarizability ${ }^{13}$ of $110.7 \mathrm{au}$, the largest calculated $\alpha(73.7 \mathrm{au})$ for $\mathrm{TiCl}_{4}$ is obtained using the $\mathrm{SBK}(\mathrm{d})^{8}$ basis set for both $\mathrm{Ti}$ and $\mathrm{Cl}$, while the smallest $\alpha(66.3 \mathrm{au})$ is obtained using the all-electron basis set: $3-21 \mathrm{G}$ for $\mathrm{Ti}$ and $3-21 \mathrm{G}^{*}$ for $\mathrm{Cl}$. In each individual comparison, ECPs always give a slightly larger $\alpha$ than a commensurate all-electron basis set and therefore a value closer to experiment. ECPs also give larger calculated $\gamma$ than all-electron basis sets, Table 1 . It is notable that
TABLE 1: ECP versus All-Electron Calculation of NLO Properties

\begin{tabular}{lccc}
\hline \multicolumn{1}{c}{ basis set } & $\begin{array}{c}\text { number of } \\
\text { basis functions }^{a}\end{array}$ & $\begin{array}{c}\alpha \\
(\mathrm{au})\end{array}$ & $\begin{array}{c}\gamma \\
(\mathrm{au})\end{array}$ \\
\hline Ti:SBK(d)/Cl:SBK(d) & 90 & 73.7 & 1378.7 \\
Ti:SBK(d)/Cl:3-21G* & 110 & 71.5 & 1187.8 \\
Ti:3-21G/Cl:SBK(d) & 85 & 68.0 & -986.6 \\
Ti:3-21G/Cl:3-21G* & 105 & 66.3 & -891.2 \\
Ti:TZV/Cl:SBK(d) & 108 & 73.0 & 1389.4 \\
Ti:TZV/Cl:3-21G* & 128 & 70.5 & 1097.2
\end{tabular}

${ }^{a}$ This is the total number of contracted Gaussians.

calculated $\gamma$ have the wrong sign when employing the allelectron double- $\zeta 3-21 \mathrm{G}$ basis set for Ti regardless of whether chlorine is described using ECPs or an all-electron basis set. This is probably due to a double- $\zeta$ VBS for titanium not being sufficiently flexible. Employing the all-electron $\mathrm{TZV}^{14}$ basis set for titanium gives very similar values compared to $\mathrm{SBK}(\mathrm{d})$, but computational times are doubled for the former. The above results show that replacing the core electrons with an effective core potential does not detract from the accuracy of calculated $\alpha$ and $\gamma$. Additionally, ECPs afford a tremendous savings in computational effort.

2. Basis Set Effects. In general, the use of an adequate basis set is the most important consideration in obtaining accurate or even reasonable NLO properties, and the basis sets required are much larger than those used in normal energy and structure calculations. ${ }^{15}$ A typical NLO basis set is often a good energy basis set augmented by several extra very diffuse $s$, $p$, and $d$ orbital functions in order to accurately describe the induced polarization of electrons. Calculation of NLO properties (particularly at the ab initio level) are often prohibitive due to the large basis sets required. In addition, previous research by Kurtz et al. ${ }^{16}$ on organic systems also suggests that basis set effects (in particular, inclusion of diffuse functions) can be larger than electron correlation effects in the accurate calculation of NLO properties. For these reasons, we first sought to isolate a basis set for further calculations that is sufficiently converged with respect to calculated NLO properties but still computationally affordable for inorganic complexes. A prototypical metal-oxo, ruthenium tetroxide $\left(\mathrm{RuO}_{4}\right)$ was chosen to illustrate basis set effects on calculated NLO properties. ECPs were used for $\mathrm{Ru}$ and $\mathrm{O}$, and different augmented valence basis sets are investigated. The geometry of $T_{d}-\mathrm{RuO}_{4}$ was optimized at the restricted Hartree-Fork (RHF) level using the SBK(d) with $\mathrm{Ru}$ having triple- $\zeta$ VBS (4d5s5p) and $\mathrm{O}$ having a double- $\zeta$-pluspolarization VBS. ${ }^{4}$ All pertinent results are shown in Table 2 and Table 3.

a. Effect of Changing the Basis Set of Oxygen. The starting basis set for this set of calculations is a compact one (90 functions) with Ru having ECPs and triple- $\zeta$ VBSs and $\mathrm{O}$ having ECPs and double- $\zeta$ valence plus d polarization basis set. This basis set, termed $\mathrm{SBK}(\mathrm{d})$, was then augmented for $\mathrm{O}$ with diffuse $\mathrm{s}, \mathrm{p}$, and d orbital functions taken from the basis set of Dykstra. ${ }^{17}$

The $\operatorname{SBK}(d)$ basis set with a more diffuse $d$ polarization function (exponent $=0.8$ ) gives an $\alpha$ value of 45.1 au, only $0.1 \mathrm{au}$ larger than $\alpha$ calculated from the basis set with a less diffuse $d$ polarization function $\left(\xi_{\mathrm{d}-\text { pol }}=0.9\right)$ suggested by Dykstra. As expected, the effect of changing the d polarization exponent on $\gamma$ is larger $(\approx 6 \%)$, but remains reasonable. Indeed, similar variation in the polarizability $(\alpha)$ and the second hyperpolarizability $(\gamma)$ can be seen in Table 2 for calculations with the same total number of basis functions.

The NLO properties $\alpha$ and $\gamma$ converge to $\approx 48$ au and $\approx 2700$ au, respectively, for the SBK(d)+Dyk-pd calculation, Table 2. 
TABLE 2: Eflects of Oxygen Basis Set Augmentation

\begin{tabular}{|c|c|c|c|c|c|c|c|c|c|}
\hline basis set & $\zeta_{\text {s-diff }}{ }^{a}$ & $\zeta_{\mathrm{pl} 1 \text {-diff }}^{b}$ & $\zeta_{\mathrm{p} 2 \text {-diff }}^{c}$ & $\zeta_{\mathrm{d}-\mathrm{pol}}{ }^{d}$ & $\zeta_{\mathrm{d} 1-\text { diff }^{e}}^{e}$ & $\zeta_{\mathrm{d} 2-\text { diff }^{f}}$ & $\begin{array}{l}\text { no. basis } \\
\text { funcs }\end{array}$ & $\alpha(\mathrm{au})$ & $\gamma(\mathrm{au})$ \\
\hline SBK(d) & & & & 0.8 & & & 90 & 45.1 & 426.7 \\
\hline SBK(d)+Dyk-pddsp & & & & 0.9 & & & 90 & 45.0 & 454.1 \\
\hline SBK $(d)+$ Dyk-pdd & 0.06 & 0.05 & & 0.9 & & & 106 & 46.2 & 2101.4 \\
\hline SBK(d)+Dyk-pdd' & 0.06 & 0.05 & & & 0.13 & & 106 & 47.3 & 2864.5 \\
\hline SBK(d)+Dyk-pdd" & 0.06 & 0.05 & & & & 0.02 & 106 & 45.8 & 2888.2 \\
\hline SBK(d)+Dyk-pd & 0.06 & 0.05 & & 0.9 & 0.13 & & 130 & 48.0 & 2655.2 \\
\hline SBK(d)+Dyk-p & 0.06 & 0.05 & & 0.9 & 0.13 & 0.02 & 154 & 48.0 & 2713.4 \\
\hline SBK $(d)+D y k$ & 0.06 & 0.05 & 0.007 & 0.9 & 0.13 & 0.02 & 166 & 48.0 & 2726.6 \\
\hline
\end{tabular}

${ }^{a}$ Diffuse $\mathrm{s}$ function. ${ }^{b}$ The first diffuse $\mathrm{p}$ function. ${ }^{c}$ The second diffuse $\mathrm{p}$ function. ${ }^{d} \mathrm{~d}$ polarization function. ${ }^{e}$ The first diffuse $\mathrm{d}$ function. ${ }^{f}$ The second diffuse $d$ function.

TABLE 3: Effects of Ruthenium Basis Set Augmentation

\begin{tabular}{|c|c|c|c|c|c|c|c|}
\hline basis set & $\zeta_{\mathrm{d} 1-\mathrm{diff}^{a}}$ & $\zeta_{\mathrm{d} 2-\text { diff }^{b}}^{b}$ & $\zeta_{\mathrm{d} 3-\text { diff }^{c}}$ & $\xi_{\text {f-pol }}^{d}$ & $\begin{array}{l}\text { no. } \\
\text { basis } \\
\text { funcs }\end{array}$ & $\begin{array}{c}\alpha \\
(\mathrm{au})\end{array}$ & $\begin{array}{c}\gamma \\
(\mathrm{au})\end{array}$ \\
\hline $\mathrm{Ru}: \mathrm{SBK}(\mathrm{d})$ & & & & & 90 & 45.1 & 426.7 \\
\hline Ru:SBK(d)+d & 0.0554 & & & & 96 & 45.8 & 1144.5 \\
\hline $\mathrm{Ru}: \mathrm{SBK}(\mathrm{d})+2 \mathrm{~d}$ & 0.0554 & 0.0277 & & & 102 & 45.9 & 1108.0 \\
\hline $\mathrm{Ru}: \mathrm{SBK}(\mathrm{d})+3 \mathrm{~d}$ & 0.0554 & 0.0277 & 0.01385 & & 108 & 45.9 & 1099.7 \\
\hline Ru:SBK(d)+f & & & & 1.235 & 100 & 44.7 & 353.3 \\
\hline
\end{tabular}

This 130 -function basis set corresponds to the addition of a diffuse $\mathrm{s}\left(\zeta_{\mathrm{s}-\text { diff }}=0.06\right)$, diffuse $\mathrm{p}\left(\zeta_{\mathrm{p} 1-\text { diff }}=0.05\right)$, and diffuse $\mathrm{d}\left(\xi_{\mathrm{d} 1-\text { diff }}=0.13\right)$ set to the $\operatorname{SBK}(\mathrm{d})$ basis set for oxygen; additionally, the d-polarization exponent used in the standard $\mathrm{SBK}(\mathrm{d})$ implementation is changed from 0.8 to 0.9 , as suggested by Dykstra. Little change is seen in $\alpha$ and $\gamma$, despite significantly longer calculation times, for the addition of a very diffuse $\mathrm{p}$ function $\left(\zeta_{\mathrm{p} 2 \text {-diff }}=0.007\right)$ and very diffuse $\mathrm{d}$ function $\left(\zeta_{\mathrm{d} 2-\text { diff }}=0.02\right)$ to the oxygen basis set, Table 2 . Hence, the SBK(d)+Dyk-pd oxygen basis set seems to afford a good compromise between computational efficiency and accuracy. The SBK(d)+Dyk-pdd' basis set, having no d polarization function compared with the $\mathrm{SBK}(\mathrm{d})+$ Dyk-pd, gives only slightly smaller $\alpha$ (47.3 au) and larger $\gamma(2864.5 \mathrm{au})$ while using $60 \%$ less computational time than the latter. This compact basis set is a viable alternative for calculation of NLO properties for larger inorganic complexes, dimers, and oligomers assuming that accurate geometries are obtained using a basis set with $d$ polarization functions on main group ligands.

b. Effect of Changing the Basis Set of Ruthenium. Compared with the effect of changing the basis set of oxygen, augmenting the basis set of ruthenium has much less of an effect on calculated NLO properties. Augmenting basis sets is generally less systematic for transition metals than lighter main group elements. The augmented $\mathrm{Ru}$ basis sets were obtained by taking the most diffuse $\mathrm{d}$ function in the standard $\mathrm{SBK}(\mathrm{d})$ valence basis set $\left(\zeta_{\mathrm{d}}=0.1108\right)$ and adding increasingly more diffuse $\mathrm{d}$ functions $\left(\xi_{\mathrm{d}}=0.1108 / N, N=2,4,8\right)$. Adding up to three more and more diffuse $\mathrm{d}$ functions causes $\alpha$ to increase only very slightly from 45.1 to 45.9 au (Table 3) and seem to converge at this value, which is lower than the converged value of 48.0 au obtained from the oxygen basis set tests, Table 2 . The $\gamma$ values are only about $40 \%$ of the approximate converged value of $2700 \mathrm{au}$ (see Table 2) and attenuate slightly when adding the second and the third diffuse $d$ functions. In Table 3 , augmenting the $\mathrm{Ru}$ basis set with an $\mathrm{f}$ polarization function ${ }^{18}$ alone gives an even smaller value for both $\alpha$ and $\gamma$. In sum, the results suggest that for $\mathrm{RuO}_{4}$ the effect of the $\mathrm{Ru}$ basis set on calculated NLO properties is substantially less than for

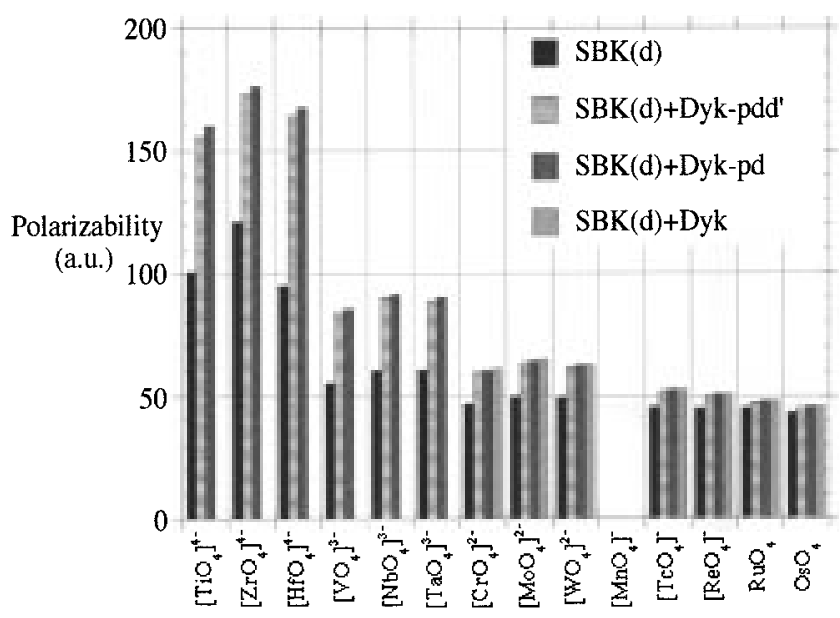

Figure 1. Polarizability of $\left[\mathrm{M}(\mathrm{O})_{4}\right]^{q-}$ using different oxygen basis sets.

oxygen. This can be explained in the following manner. The large positive formal charge on $\mathrm{Ru}(+8)$ suggests that its contribution to the overall $\alpha$ and $\gamma$ is less important than the anionic oxide ligands at the periphery of the complex. This result is expected to be applicable to the large majority of metalate and related complexes since these tend to have the metal in its largest formal oxidation state.

3. NLO Properties for Transition Metal Metalates, $\left[\mathrm{MO}_{4}\right]^{q-}$. Having identified suitable basis sets, we further employ them to investigate NLO properties for a series of metalate complexes $(\mathbf{1}),\left[\mathrm{MO}_{4}\right]^{q-}$. The results are summarized in Figures 1 and 2. Metalates have a tetrahedral geometry; thus, the first hyperpolarizability $(\beta)$ is zero.

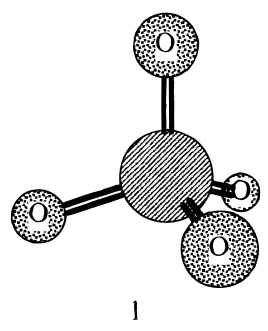

Inspection of Figures 1 and 2 indicates that trends in polarizability and hyperpolarizability of metalates are not inordinately sensitive to oxygen basis set. The SBK(d) (90 basis functions), SBK(d)+Dyk-pdd' (106 basis functions), SBK(d)+Dyk-pd (130 basis functions), and SBK(d)+Dyk (166 basis functions) calculations were studied, and similar trends as a function of metal were seen. The $\operatorname{SBK}(d)$ basis set is not sufficient to give accurate NLO properties (values obtained are too small), Figures 1 and 2. Theagreement of both $\alpha$ and $\gamma$ 


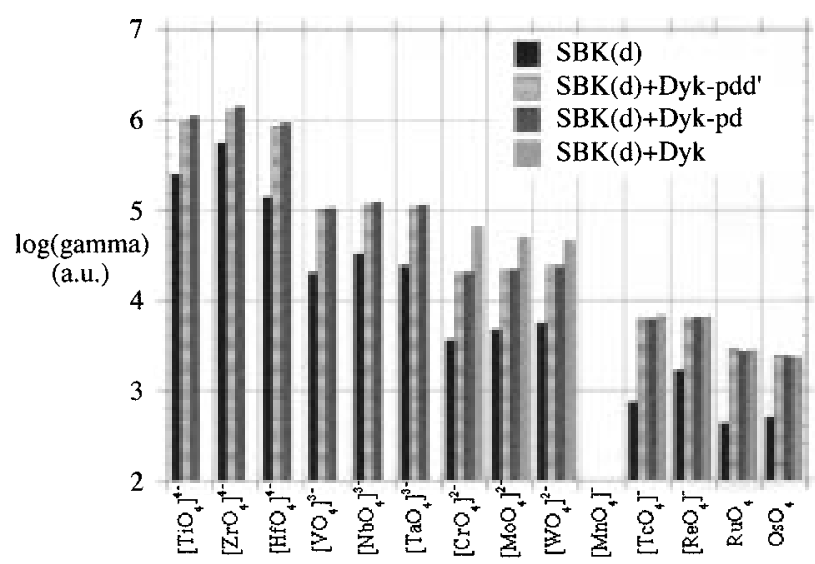

Figure 2. $\log (\gamma)$ of $\left[\mathrm{M}(\mathrm{O})_{4}\right]^{q-}$ using different oxygen basis sets.

obtained from the moderately augmented SBK(d)+Dyk-pdd' and $\mathrm{SBK}(\mathrm{d})+$ Dyk-pd basis sets supports the $\mathrm{RuO}_{4}$ results with respect to convergence of calculated NLO properties. Basis set effects in the calculation of $\alpha$ become less significant as the charge on the complex becomes less negative, Figure 1. For $\gamma$, the basis set dependence is similar across the transition series, Figure 2.

The polarizability and second hyperpolarizability decrease from left to right across the transition series, Figures 1 and 2. This is probably due to the lower overall negative charge (culminating in neutral $\mathrm{RuO}_{4}$ and $\mathrm{OsO}_{4}$ ) as the metal formal oxidation state increases. An interesting trend is that within a triad the complex with the second transition series metal has the largest calculated $\alpha$, Figure 1. The difference between second and third series metalates is much smaller than that between these and the first series metalates. Trends in $\alpha$ are in rough agreement with the variation in calculated $\mathrm{M}-\mathrm{O}$ bond lengths ${ }^{4}$ of $\left[\mathrm{MO}_{4}\right]^{q-}$. (The correlation of $\alpha$ calculated from the SBK(d)+Dyk-pd basis set versus $\mathrm{M}-\mathrm{O}$ bond length gives $\alpha$ $(\mathrm{au})=318.0 \times \mathrm{M}-\mathrm{O}(\AA)-479.5 ; R=0.90$. Similarly, log$(\gamma)(\mathrm{au})=6.30 \times \mathrm{M}-\mathrm{O}(\AA)-6.58 ; R=0.88$. $)$ This trend is a reflection of the structure-property relationships for polarizability, because for the same number of valence electrons the larger the molecular size (as indicated by a longer $\mathrm{M}-\mathrm{O}$ bond), the more diffuse the electron distribution of the metalate will be, and hence the larger the polarizability. But there are also some exceptions to this generalization. The third transition series metalates of groups VIB, VIIB, and VIII $\left(\mathrm{WO}_{4}{ }^{2-}, \mathrm{ReO}_{4}{ }^{-}\right.$, and $\mathrm{OsO}_{4}$ ) have slightly smaller polarizability than their analogues in the second series though their $\mathrm{M}-\mathrm{O}$ bond lengths are all slightly larger.

Another interesting trend is that, in each group, while the similarities of both $\alpha$ and $\gamma$ for the second and the third series $\left[\mathrm{MO}_{4}\right]^{q-}$ are consistent with bond length trends, the relative differences between them and the corresponding first series $\left[\mathrm{MO}_{4}\right]^{q-}$ are much smaller than expected given the larger difference in relevant $\mathrm{M}-\mathrm{O}$ bond lengths. $\mathrm{MnO}_{4}{ }^{-}$has the shortest $\mathrm{M}-\mathrm{O}$ bond length among all the 14 calculated $\left[\mathrm{MO}_{4}\right]^{q-}$. Thus the smallest $\alpha$ value is expected for $\mathrm{MnO}_{4}{ }^{-}$. Unfortunately, $\mathrm{NLO}$ data for $\mathrm{MnO}_{4}{ }^{-}$could not be obtained due to the convergence failure of the TDHF calculation for this complex.

4. Geometry Effects. The experimental geometry (gasphase electron diffraction $\left.{ }^{6}\right)$ and polarizability of $\mathrm{OsO}_{4}(\alpha=55.1$ au in liquid phase ${ }^{13}$ ) are available. Thus, $T_{d}-\mathrm{OsO}_{4}$ is used to evaluate geometry effects on calculated NLO properties. It is seen from the results in Table 4 that agreement between optimized and experimental geometries is quite good with the
TABLE 4: Geometry Effects on Calculated NLO Values for $\mathrm{OsO}_{4}$

\begin{tabular}{|c|c|c|c|c|c|c|}
\hline geometry/NLO ${ }^{a}$ & $\begin{array}{c}\mathrm{Os}-\mathrm{O} \\
(\AA)\end{array}$ & $\begin{array}{c}\Delta \% \\
(r)\end{array}$ & $\begin{array}{c}\alpha \\
(\mathrm{au})\end{array}$ & $\begin{array}{l}\Delta \% \\
(\alpha)\end{array}$ & $\begin{array}{c}\gamma \\
(\mathrm{au})\end{array}$ & $\begin{array}{l}\Delta \% \\
(\gamma)\end{array}$ \\
\hline & & 0 & 455 & 0 & 2277.1 & 0 \\
\hline & & 0.1 & 45 & 0.3 & & \\
\hline$x p t / S B K(d)+D y k$ & 1.712 & 3 & 48.5 & 7 & 2512.8 & 10 \\
\hline
\end{tabular}

${ }^{a}$ The geometry is optimized at the first basis set indicated or obtained from experiment; the second basis set is that used for NLO property calculation.

difference of only $3 \%$ (this level of agreement is seen for all $\left[\mathrm{MO}_{4}\right]^{q-}$ complexes $\left.^{4}\right)$. There is almost no difference between the Os-O bond length optimized using the SBK(d) and SBK(d) + Dyk basis sets, Table 4 , and hence only a slight $(0.3 \%)$ improvement in calculated $\alpha$ and $\gamma$ using the latter. Using the experimental geometry of $\mathrm{OsO}_{4}$ shows a more significant change in $\alpha$ (7\% increase) and $\gamma$ (10\% increase). The calculated $\alpha$ using the largest basis set (SBK(d)+Dyk) for geometry optimization and property calculation is about $83 \%$ of the experimental value. The calculated $\alpha$ using the experimental geometry and the SBK(d)+Dyk basis set is about $88 \%$ of experiment. These data suggest that geometry and basis set effects have reached their limits with respect to further approach of calculated values to experimental NLO properties.

\section{Conclusions}

A computational investigation of the nonlinear optical properties of inorganic complexes is reported. Several important results have been reached, the most important of which are summarized below.

(1) Effective core potentials have been shown to be competent for the calculation of NLO properties by comparison with allelectron calculations. The polarizability $(\alpha)$ and second hyperpolarizability $(\gamma)$ were studied here since tetrahedral molecules have a first hyperpolarizability $(\beta)$ equal to zero. In these comparisons it is important that the description of the valence orbitals be commensurate with each other.

(2) Basis set effects are important for the calculation of the NLO properties. Taking a valence basis set suitable for geometry optimization of transition metal complexes (i.e., triple- $\zeta$ for TMs and double- $\zeta$-plus-polarization for MG elements) and augmenting the MG basis set with diffuse functions of angular momentum $l=0,1$, and 2 provided the best compromise between speed and accuracy.

(3) Basis set effects seem to converge with the addition of diffuse s, p, and d functions to the SBK(d) basis set, which has been shown to be reliable for geometry prediction. This research suggests that $d$ polarization functions are less important than diffuse $d$ functions in the calculation of NLO properties, and hence basis sets like SBK(d)+Dyk-pdd' may be useful for the study of larger inorganic complexes as long as basis sets like $\mathrm{SBK}(\mathrm{d})$ are used to calculate geometries when these cannot be obtained or estimated from experimental data.

(4) Basis set effects are found to be less important for the central transition metal than the surrounding ligands. To some extent this is expected given the large formal oxidation states on these metals and the substantial anionic character of the ligands. It will be of interest to assess TM basis set effects for complexes with metals in lower oxidation states.

(5) Both $\alpha$ and $\gamma$ decrease for $\left[\mathrm{MO}_{4}\right]^{q-}$ toward the right in the transition series. The $\alpha$ values for the second series $\left[\mathrm{MO}_{4}\right]^{q-}$ are the largest among the three metals in a triad. For groups 
IVB and VB complexes with larger charge $(-4$ and -3 , respectively), the second series $\left[\mathrm{MO}_{4}\right]^{q-}$ have the largest $\gamma$ values, while for groups VIB, VIIB, and VIII with less charge $(-2,-1$, and 0 , respectively), the third series metalates have the largest $\gamma$. The relative difference in both $\alpha$ and $\gamma$ values among the three series in the same group is much smaller than between different groups. Overall, variations in the calculated values of NLO properties correlate with $\mathrm{M}-\mathrm{O}$ bond lengths.

The limits observed in augmenting basis sets and from changing the geometry suggest the need to include electron correlation in the calculation of NLO properties. Such research may necessitate a change from TDHF to finite-field (FF) methods. Another important effect, that of the environment, must also be considered by calculating dimers and larger oligomers in the gas phase, modeling solvent effects, and modeling solid-phase materials. NLO properties of transition metal-sulfidos, selenidos, and telluridos can also be calculated to give more data to better understand the relations between structure and the NLO properties. The present research is encouraging with respect to the further use of computational chemistry for modeling the NLO properties of transition metal complexes. Such studies are now under way in our lab.

Acknowledgment. T.R.C. acknowledges support of this research through Grant CHE-9614346 from the National Science Foundation. The authors also thank the NSF centers (Cornell Theory Center and National Center for Supercomputing Applications) for grants of supercomputer time. Additionally, H.A.K. and T.R.C. acknowledge the generous support of the NSF for computational chemistry research at the University of Memphis through Grants STI-9602656 (from the Academic Research Infrastructure program) and CHE-9708517 (from the Chemical Research Instrumentation and Facilities program).

\section{References and Notes}

(1) E-mail: tcundari@memphis.edu.

(2) Marder, S. R., Sohn, J. E., Stucky, G. D., Eds. ACS Symposium Series 455; American Chemical Society: Washington, DC, 1991.

(3) Long, N. J. Angew. Chem Int., Ed. Engl. 1995, 34, 21.

(4) Benson, M. T.; Cundari, T. R.; Lim, S. J.; Nguyen, H. D.; PierceBeaver, K. J. Am. Chem. Soc. 1994, 116, 3955.

(5) Cundari, T. R.; Raby, P. D. J. Phys. Chem. A 1997, 101, 5783.

(6) Nugent, W. A.; Mayer, J. M. Metal-Ligand Multiple Bonds; Wiley: New York, 1988.

(7) (a) Kanis, D. R.; Ratner, M. A.; Marks, T. J. J. Am. Chem. Soc. 1990, 112, 8203. (b) Kanis, D. R.; Lacroix, P. G.; Ratner, M. A.; Marks, T. J. J. Am. Chem. Soc. 1994, 116, 10089. (c) Kanis, D. R.; Ratner, M. A.; Marks, T. J. J. Am. Chem. Soc. 1992, 114, 10338. (d) Kanis, D. R.; Ratner, M. A.; Marks, T. J. Chem. Rev. 1994, 94, 195.

(8) Krauss, M.; Stevens, W. J.; Basch, H.; Jasien, P. G. Can. J. Chem. 1992, $70,612$.

(9) Cundari, T. R.; Gordon, M. S. Coord. Chem. Rev. 1996, 147, 87. (10) Kurtz, H. A.; Dudis, D. S. Reviews in Computational Chemistry; Boyd, D. B., Lipkowski, K. B., Eds., in press.

(11) Schmidt, M. W.; Baldridge, K. K.; Boatz, J. A.; Jensen, J. H.; Koseki, S.; Matsunaga, N.; Gordon, M. S.; Nguyen, K. A.; Su, S.; Windus, T. L.; Elbert, S. T.; Montgomery, J.; Dupuis, M. J. Comput. Chem. 1993, $14,1347$.

(12) Korambath, P.; Kurtz, H. A. In Theoretical and Computational Modeling of NLO and Electronic Materials; Karna, S., Yates, A. T., Eds.; American Chemical Society: Washington, DC, 1996.

(13) Handbook of Chemistry and Physics, 78th ed.; CRC Press: Boca Raton, 1997-1998; pp 10-204.

(14) (a) Dunning, T. H. J. Chem. Phys. 1971, 55, 716. (b) Rappe, A K.; Smedley, T. A.; Goddard, W. A., III. J. Phys. Chem. 1981, 85, 2607. (15) Feller, D.; Davidson, E. R. In Reviews in Computational Chemistry; Lipkowitz, K. B., Boyd, D. B., Eds.; VCH Publishers: New York, 1990; Vol. 1, p 1.

(16) Chen, S.; Kurtz, H. A. J. Mol. Struct. (THEOCHEM) 1996, 388 , 79

(17) Dykstra, C. E.; Liu, S.-Y.; Malik, D. J. Adv. Chem. Phys. 1989, $75,37$.

(18) Ehlers, A. W.; Böhme, M.; Dapprich, S.; Gobbi, A.; Höllwarth, A.; Jonas, V.; Köhler, K. F.; Stegmann, R.; Veldkamp, A.; Frenking, G. Chem. Phys. Lett. 1993, 208, 111. 